FORMATION Formation emploi

Revue française de sciences sociales

93 | janvier-mars 2006

Au-delà du genre, l'insertion

\title{
Au fur et à mesure qu'elles se construisent, les trajectoires professionnelles divergent entre les sexes : l'exemple belge
}

Occupational trajectories gradually diverge with time between the sexes: the case of Belgium

Berufliche Werdegänge von Frauen und Männern in Belgien

\section{Claire Gavray}

\section{(2) OpenEdition}

\section{Journals}

Édition électronique

URL : http://journals.openedition.org/formationemploi/2370

DOI : 10.4000/formationemploi.2370

ISSN : 2107-0946

Éditeur

La Documentation française

\section{Édition imprimée}

Date de publication : 1 mars 2006

Pagination : 49-65

ISSN : 0759-6340

\section{Référence électronique}

Claire Gavray, «Au fur et à mesure qu'elles se construisent, les trajectoires professionnelles divergent entre les sexes : l'exemple belge », Formation emploi [En ligne], 93 I janvier-mars 2006, mis en ligne le 03 décembre 2009, consulté le 30 octobre 2020. URL : http://journals.openedition.org/

formationemploi/2370; DOI : https://doi.org/10.4000/formationemploi.2370 


\section{Au fur et à mesure qu'elles se construisent, les trajectoires professionnelles divergent entre les sexes : l'exemple belge}

Par Claire Gavray*

En tout début de carrière, le sort des femmes et des hommes est assez proche. Les rapports sociaux de sexe pèsent ensuite différemment sur les choix personnels, familiaux ou parentaux et dans le domaine de l'emploi. Ainsi avec le temps, les écarts professionnels se creusent entre les sexes et groupes sociaux.

Est-on parvenu à une égalisation des statuts et des ressources professionnelles des jeunes hommes et des jeunes femmes accédant au marché de l'emploi ? Si un écart significatif persiste, dans quelle mesure fautil l'attribuer à des facteurs liés à l'offre ou à la demande de travail? Quels nouveaux éléments de compréhension nous apportent les données longitudinales pour étudier les débuts de trajectoires d'emploi ? Ce sont les questions auxquelles nous tentons de répondre dans cet article à partir des données du Panel Study on Belgian Household (volet belge de l'European Community Household Panel).

Des recherches récentes témoignent d'une égalisation progressive des positions professionnelles, communément attribuée au rattrapage des niveaux de scolarité féminins (Baudelot et Glaude, 1989) ainsi qu'à une mixité grandissante de la culture jeune (valeurs, attitudes et projets). Même si ce rapprochement se
Claire Gavray est docteure en sociologie, chercheuse et enseignante à l'université de Liège (Belgique) au sein du département "Personne et Société " de la faculté de psychologie et des sciences de l'éducation - Service d'étude du développement psychosocial - ainsi qu'au sein de l'Institut des sciences humaines et sociales. Ses travaux portent essentiellement sur l'étude des trajectoires de vie dans l'articulation de leurs différentes dimensions (socio-économiques, professionnelles, psychosociales et relationnelles, démographiques, familiales), sur les risques de précarisation et de marginalisation ainsi que sur le genre. Parmi ses publications récentes: Gavray C. (2004a), "Les premières années d'insertion professionnelle en liaison aux autres engagements et à la lumière du genre », in Doutrelepont R., Mortelmans D., Casman 
M.-T., Onze ans de vie en Belgique, Gent, Academia Press, pp. 295-324. Gavray C. (2004b), "Engagements familiaux et professionnels : regard sur les projets des jeunes hommes et femmes en fin de formation scolaire », in Istace E., Laffut M., Plasman R., Ruyters C., Sphères privées et professionnelles : vers une recomposition des rôles et des actions, de Boeck, coll. "Économie, Société et Région», pp. 199-220.

confirme, cette tendance à l'égalisation persiste-t-elle dans la durée au sein de la cohorte étudiée ? En effet, un fossé pourrait se creuser à nouveau au désavantage des femmes au fur et à mesure que les individus franchissent les différentes étapes de la vie adulte (engagements professionnels, mise en couple, obligations parentales...). Ainsi, certaines études montrent la résistance des mécanismes de répartition traditionnelle de la charge de travail (Barrere-Maurisson, 1992; Perrot et al., 1999) et des responsabilités familiales (Leridon et Villeneuve-Gokalp, 1994). Cela reste vrai malgré la variété toujours plus grande des configurations familiales et des styles de vie (Galland 1995). D'autres recherches prouvent qu'une offre d'emploi différenciée continue à être adressée aux deux groupes sexués, y compris pour un même niveau de diplôme. Cette réalité engendrerait des conséquences individuelles discriminatoires en matière de statut (Maruani, 1996), de mobilité de carrière (Chapoulie, 2000) ou de construction de la carrière (Wijn et Pruyer, 2000). Malgré une diversification des modèles d'insertion professionnelle (Marry, 1996) en début d'âge adulte et ce, dans les deux groupes sexués, les jeunes femmes, plus que les jeunes hommes, payent un lourd tribu (Nicole-Drancourt, 1990) au mouvement de flexibilisation et de fragilisation de l'insertion professionnelle (Bellens, 1996; Rose, 1998) et au phénomène plus large de la dualisation du marché du travail et de l'emploi pressenti par Piore (1978) ou Favereau (1989). Elles se retrouvent finalement les plus concernées par le sous-emploi, cela principalement via l'emploi à temps partiel qui leur est réservé, surtout aux moins qualifiées d'entre elles (Kergoat, 1984) mais pas exclusivement. Ainsi, les recherches récentes confirment unanimement qu'au sein des pays européens, le rôle du diplôme reste plus crucial pour l'entrée et le maintien en emploi du groupe féminin que du groupe masculin
(Establet, 1988). Le niveau de diplôme influence également l'adoption de stratégies d'autonomie payantes (Commaille, 1992), par exemple en matière d'articulation temporelle des engagements familiaux et professionnels (Blöss et al., 1994 ; Terrail, 1992).

Comment approcher et comprendre le parcours professionnel des jeunes gens et des jeunes femmes?

Notre investigation s'appuie sur les différents éléments issus de la recherche récente. Nous faisons l'hypothèse que les rapports sociaux de sexe, toujours à l'œuvre tant dans la construction de l'offre de travail que de l'offre d'emploi, viendraient alimenter les inégalités au sein des jeunes générations, cela même si on peut vérifier par ailleurs une plus grande liberté de ces dernières à diversifier les dimensions qu'elles veulent donner à leur existence. La théorie du genre permet selon nous de comprendre, sous des aspects renouvelés, les nouveaux parcours de vie et d'emploi. L'essentiel de cette approche ne réside pas dans la constatation de différences entre les sexes mais dans l'identification des systèmes de relations sexuées, formelles ou informelles, entraînant des inégalités en matière de négociation et dans la répartition des ressources et des positions.

Les résultats présentés comparent le début de trajectoire professionnelle de jeunes hommes et de jeunes femmes. Nos analyses concernent un sous-échantillon de jeunes gens et de jeunes filles suivis par l'enquête durant les cinq ans qui suivent la sortie de leur scolarité. La banque de données utilisée autorise un suivi des personnes dans le temps. Il permet également de visualiser à la fois les articulations entre les différents engagements dans la durée (familiaux, professionnels) et la corrélation entre les caractéristiques et positions individuelles avec celles du partenaire ou du conjoint éventuel.

127 sujets sont concernés par l'analyse, dont $55 \%$ de jeunes hommes. L'âge moyen de fin d'études est de 23 ans et trois mois. La taille restreinte de ce sous-échantillon nous impose d'emblée certaines limites quant au nombre de modalités des variables à utiliser, ainsi que certaines précautions dans l'analyse des résultats. Par exemple, nous classerons souvent les sujets en deux groupes, selon qu'ils possèdent ou non un diplôme supérieur à celui de l'enseignement secondaire.

Dans une première partie de l'article, nous rendrons compte des positions occupées à deux moments du temps : respectivement en avril 1995, 1996, 1997, soit près d'un an et demi après l'arrêt de la scolarité, 


\section{Encadré 1 \\ Le volet belge de l'European Community Household Panel}

La banque de données utilisée, le Panel Study on Belgian Households, (PSBH), comprend les données de onze vagues d'enquêtes effectuées de 1992 à 2002 auprès d'un échantillon représentatif et "vivant » de ménages et de personnes (c'est-à-dire qui inclut progressivement les nouveaux membres, suit les sujets qui quittent leur ménage d'origine au profit d'un nouveau... Pour plus de détails sur la méthodologie utilisée et le contenu de l'enquête, consulter www.ulg.ac.be/psbh). L'échantillon de départ comportait 4438 ménages, 8741 adultes d'au moins seize ans et 2594 sujets plus jeunes. Les informations relatives au ménage (logement...) et aux enfants ont été recueillies auprès de la personne de référence du ménage. De plus, chaque membre du ménage de plus de seize ans a été interrogé personnellement une fois par an sur sa situation familiale, son statut socioprofessionnel, ses conditions de travail et d'emploi, ses revenus personnels, les formations et enseignements suivis, ses loisirs, sa santé physique et psychologique, ses relations sociales et ses engagements citoyens...

Le caractère longitudinal et généraliste de l'enquête et de l'échantillon permet ainsi d'observer et d'analyser l'articulation entre les diverses dimensions de la vie trop souvent séparées, de mettre en regard les caractéristiques des différents membres d'un même ménage. En contrepartie, les opportunités d'analyser une problématique pointue ou une sous-population très ciblée restent réduites.

Le PSBH permet de suivre la trajectoire professionnelle des mêmes individus dans la durée et d'examiner le cursus d'insertion de jeunes gens ayant arrêté leur scolarité à la même période. Ainsi, nous avons construit un sous-échantillon de sujets masculins et féminins ayant terminé leur formation initiale $\left({ }^{*}\right)$ secondaire ou supérieure en 1993, 1994 ou 1995. On envisage ici la formation scolaire débutée dans l'enfance. Après six ans d'école primaire, celle-ci se poursuit dans l'enseignement secondaire six autres années, parfois sept pour ceux qui ne poursuivent pas au-delà du secondaire. L'enseignement supérieur, quant à lui, et à l'époque de l'enquête, est divisé entre une filière de type court et une de type long. Tant dans le secondaire que dans le supérieur, on trouve des études théoriques et générales et d'autres faisant une large part à la formation pratique, au stage et à l'apprentissage en entreprise. On n'envisage pas ici le cas des personnes ayant repris des études supérieures après avoir quitté, momentanément et pour l'une ou l'autre raison, le statut d'étudiant.

(*) : diplôme en main ou non.

ensuite en avril 1998, 1999 et 2000, soit trois ans après le premier bilan et au total un peu moins de cinq ans après la fin de la scolarité (avec ou sans diplôme ou certification finale). Notons que nous disposons de différentes données concernant la période comprise entre ces deux temps de mesure, par exemple le nombre de mois passés en emploi et hors emploi.

Nous discuterons d'abord de la question de l'impact du niveau de diplôme sur la position occupée. Dans une seconde partie, nous comparerons l'articulation entre les situations démographiques et d'emploi dans le groupe masculin et féminin, la position théorique la plus courante avancée pour expliquer la moindre qualité des trajectoires professionnelles des femmes étant la charge familiale reposant sur ces dernières, ou encore le déficit d'implication ou d'ambition dont elles peuvent faire preuve. Nous discuterons ce point. La troisième étape étudiera la construction des trajectoires d'emploi en regard à d'autres facteurs, relatifs cette fois-ci à l'offre d'emploi et à l'enchaînement des statuts et des transitions.

\section{LES FEMMES SONT VITE DISTANCÉES}

Les jeunes femmes ont, en début de carrière, des positions proches de leurs homologues masculins; ensuite, les écarts se creusent. Et cela reste vrai même pour les plus diplômées. 


\section{Des positions professionnelles proches un an et demi après l'arrêt de la scolarité}

Les données confirment des difficultés d'insertion professionnelle des jeunes sortants. Plus d'un cinquième recherchent un emploi au temps 1 , soit vingt mois après l'arrêt de la scolarité. De prime abord, les positions des jeunes hommes et des jeunes femmes face à l'emploi sont proches. Ainsi, au premier moment de mesure, il n'y a pas de probabilité significativement différente d'accès à l'emploi ou de risque de chômage pour les jeunes hommes et les jeunes femmes, pas plus que de différence en matière de détention d'un emploi à durée indéterminée. Néanmoins, une différence significative s'observe entre les sexes au niveau du temps de travail contractuel. Ainsi, $86 \%$ des salariés masculins contre $61 \%$ des salariées occupent au temps 1 un emploi à temps plein et ce, sans que l'on puisse expliquer l'acceptation d'un poste à temps partiel ${ }^{1}$ comme le résultat de contraintes familiales ou comme un choix personnel. Les réponses des sujets à cette question en attestent. Notons que près de $30 \%$ des sujets féminins du groupe le plus diplômé (supérieur de type long) sont également significativement concernés par cette modalité d'emploi. En outre, $61 \%$ des hommes en activité occupent un emploi classiquement décrit comme typique (c'est-à-dire à la fois à temps plein et à durée indéterminée), contre $27 \%$ des femmes.

${ }^{1}$ ou plus exactement un poste où le temps de travail prévu est inférieur à 30 heures par semaine. En effet, dès la troisième vague d'enquête PSBH, les travailleurs ne sont plus appelés à s'auto-classer en tant que travailleurs à temps plein ou à temps partiel.

\section{Les écarts de position augmentent cinq ans après la fin de la scolarité}

Trois ans après la première mesure (soit cinq ans après l'arrêt des études), trois quarts des sujets masculins et féminins, y compris ceux hors emploi à ce moment, ont désormais connu au moins une expérience professionnelle de plus de 15 heures hebdomadaires. Le pourcentage des jeunes effectivement en emploi a augmenté pour concerner désormais près de neuf jeunes sur dix. La comparaison entre les sexes révèle des inégalités sexuées précédemment non significatives, et cela au désavantage des femmes. Presque tous les jeunes hommes effectuent à ce moment un travail rémunéré (contre trois quarts des femmes). À l'inverse, le pourcentage de femmes hors emploi ou chômeuses, y compris selon les critères exigeants du $\mathrm{BIT}^{2}$, est significativement supérieur à celui des hommes. Le tableau 1 indique que, selon les cas, les écarts hommes/femmes sont significatifs au seuil de $1 \%$.

$\mathrm{Au}$ niveau de la qualité de l'emploi occupé, de nouvelles inégalités apparaissent, tandis que d'autres, déjà mises en lumière, se sont creusées. Ainsi, même si la proportion de travailleurs en emploi typique a bien augmenté entre les deux moments de mesure, désormais plus de quatre salariés masculins sur cinq détiennent un contrat à durée indéterminée contre

\footnotetext{
${ }^{2}$ Pour les années considérées, le BIT retenait comme chômeur toute personne qui, durant une semaine de référence déterminée, n'a pas travaillé, même pas une heure, était disponible pour commencer à travailler dans un délai de deux semaines et avait entrepris des démarches spécifiques de recherche d'emploi au cours des 4 semaines précédant la semaine de référence.
}

Tableau 1

\section{Emploi et chômage BIT(*) selon les phases d'insertion}

\begin{tabular}{|l|c|c|c|c|c|c|}
\hline & \multicolumn{2}{|c|}{ +/- un an et demi après la fin des études (temps 1) } & \multicolumn{3}{|c|}{ cinq ans après la fin des études (temps 2) } \\
\hline & Hommes & Femmes & Total & Hommes & Femmes & Total \\
\hline En emploi & $80 \%$ & $71 \%$ & $72 \%$ & $96 \%$ & $74 \%$ & $87 \%$ \\
\hline & \multicolumn{2}{|c|}{ différence non significative } & & différence significative & \\
\hline Chômeurs BIT & $18 \%$ & $26 \%$ & $21 \%$ & $4 \%$ & $18 \%$ & $10 \%$ \\
\hline
\end{tabular}

PSBH, sous-échantillon total

Seuil de significativité à $1 \%$.

$(*)$ : Bureau international du travail. 
Tableau 2

\section{Types de contrats de travail selon les phases d'insertion}

\begin{tabular}{|c|c|c|c|c|c|c|}
\hline \multirow[t]{2}{*}{ Type de contrat } & \multicolumn{3}{|c|}{$\begin{array}{l}+/ \text { - un an et demi après la fin des études } \\
\text { (temps 1) }\end{array}$} & \multicolumn{3}{|c|}{$\begin{array}{l}\text { cinq ans après la fin des études } \\
\text { (temps 2) }\end{array}$} \\
\hline & Salariés & Salariées & Total & Salariés & Salariées & Total \\
\hline \multirow[t]{2}{*}{ à durée indéterminée } & $60 \%$ & $48 \%$ & $55 \%$ & $82 \%$ & $63 \%$ & $74 \%$ \\
\hline & \multicolumn{2}{|c|}{ différence non significative } & & \multicolumn{2}{|c|}{ différence significative } & \\
\hline \multirow[t]{2}{*}{ à temps partiel (- $30 \mathrm{H}$ /sem.) } & $14 \%$ & $39 \%$ & $24 \%$ & $10 \%$ & $41 \%$ & $20 \%$ \\
\hline & \multicolumn{2}{|c|}{ différence significative } & & \multicolumn{2}{|c|}{ différence significative } & \\
\hline \multirow[t]{2}{*}{ à temps partiel et en CDD } & $9 \%$ & $18 \%$ & $13 \%$ & $2 \%$ & $21 \%$ & $9 \%$ \\
\hline & \multicolumn{2}{|c|}{ différence significative } & & \multicolumn{2}{|c|}{ différence significative } & \\
\hline
\end{tabular}

PSBH, salariés du sous-échantillon

Seuil de significativité inférieur à $1 \%$

moins de deux salariées sur trois (voir tableau 2). Quant au contrat à temps partiel, il se confirme comme une modalité d'emploi spécifiquement féminine. Par ailleurs, il n'est pas réparti selon les mêmes critères personnels au sein des deux groupes sexués : chez les hommes, il constitue soit une passerelle vers un emploi indépendant, soit une position de transition vers un emploi à temps plein. En seconde période, on retrouve presque vingt fois moins de travailleurs masculins que féminins occupant un emploi à durée déterminée et dont la durée hebdomadaire n'atteint pas 30 heures. Désormais, plus des trois quarts des travailleurs masculins (78\%) occupent un emploi typique (à temps plein et à durée indéterminée) contre moins de la moitié des travailleuses (46\%).

Nous reviendrons plus loin sur la question de l'enchaînement de statuts et sur la dimension temporelle de l'insertion professionnelle, les données utilisées nous permettant de suivre les personnes dans le temps. Les résultats laissent peu de doute sur certaines dynamiques particulièrement fragilisantes qui ne frappent pas au hasard les jeunes travailleurs.

\section{L'impact crucial du niveau de diplôme se vérifie pour les jeunes femmes}

Une meilleure réussite scolaire des filles se confirme au niveau de l'échantillon; en effet, les femmes, dans une large proportion, détiennent, plus que les hommes, un diplôme de l'enseignement supérieur court ou long?3.

\begin{abstract}
${ }^{3}$ Notons que si nous connaissons le plus haut niveau d'études suivi ou atteint (secondaire ou supérieur de type court ou long), nous ne disposons pas au départ de l'enquête d'information précise sur le type d'études suivies, ni sur le métier auquel ces dernières préparent. Nous pouvons ainsi confirmer une surreprésentation des filles dans des études courtes (souvent 3 ans après le secondaire) mais nous ne sommes pas en mesure de discuter l'impact des orientations scolaires et professionnelles différenciées prises par les deux groupes sexués.
\end{abstract}

Tableau 3

Niveau du dernier type d'enseignement fréquenté et du plus haut niveau de diplôme atteint

\begin{tabular}{|l|c|c|c|c|}
\hline & Niveau du dernier enseignement fréquenté & \multicolumn{2}{|c|}{ Niveau du plus haut diplôme atteint } \\
\hline & Hommes & Femmes & Hommes & Femmes \\
\hline Enseignement secondaire & $20 \%$ & $30 \%$ & $69 \%$ & $51 \%$ \\
\hline Enseignement supérieur type court & $46 \%$ & $31 \%$ & $14 \%$ & $29 \%$ \\
\hline $\begin{array}{l}\text { Enseignement supérieur } \\
\text { type long + universitaire }\end{array}$ & $34 \%$ & $39 \%$ & $17 \%$ & $20 \%$ \\
\hline & \multicolumn{2}{|c|}{ différence sexuée non significative } & différence sexuée significative \\
\hline
\end{tabular}


Beaucoup plus que pour les jeunes hommes, le diplôme représente un laissez-passer indispensable pour les femmes désireuses de s'inscrire durablement dans 1'emploi : $50 \%$ des jeunes femmes diplômées au maximum de l'enseignement secondaire se retrouvent hors emploi au temps 2, contre seulement $13 \%$ des diplômées de l'enseignement supérieur. Néanmoins, parallèlement, un niveau de diplôme élevé n'empêche pas significativement les femmes d'occuper un emploi à temps partiel ou atypique. Le pourcentage de travailleuses concernées de ce groupe reste assez stable par rapport au temps 1 . Moins de $5 \%$ des hommes diplômés du supérieur connaissent ce statut de travail et c'est ici surtout le cas de jeunes hommes envisageant une transition vers l'emploi indépendant. Entre les deux moments d'observation, le temps de travail hebdomadaire déclaré par les sujets salariés n'a pas beaucoup évolué : 41 heures pour les hommes, tandis qu'il passe de 35 à 33 heures pour les femmes. Les enjeux du temps se précisent. Les heures de travail décollent dans le groupe masculin et se tassent dans le groupe féminin, $y$ compris parmi les femmes plus diplômées et très majoritairement en emploi.

Quant au type d'horaire proprement dit, aux deux moments d'observation, un tiers des travailleurs masculins mentionnent une situation où le nombre d'heures de travail et le moment de la semaine ou de la journée où ces dernières sont effectuées varient de semaine en semaine. Cette modalité de travail exigeant une disponibilité extrême ne vaut plus que pour une travailleuse sur dix au temps 2 , proportionnellement deux fois moins qu'au temps 1 . Le travail de nuit et de fin de semaine concerne au masculin une plus grande diversité de qualifications et de secteurs d'activités qu'au féminin. Les travailleuses les plus qualifiées, d'un côté, et les moins qualifiées, de l'autre, exerçant leur profession dans les secteurs du commerce, des services et de la santé sont les plus exposées à l'imprévisibilité des horaires.

Parallèlement, au temps 1, et alors que, rappelons-le, le niveau de certification est favorable au groupe féminin, trois fois plus de jeunes gens que de jeunes femmes se sont classés parmi les employés qualifiés ou cadres, les femmes se concentrant majoritairement dans la catégorie de « simple employé », y compris si on limite l'analyse au groupe des plus diplômés. Au total, comme prévu (Gavray, 2003), elles ont moins intériorisé les opportunités de carrière et de promo- tion auxquelles elles pourraient prétendre ; elles estiment plus souvent occuper un poste pour lequel elles sont surqualifiées.

Dès le départ, les jeunes femmes se concentrent majoritairement dans la catégorie des bas revenus du travail. Mais surtout, elles s'y enlisent plus que les jeunes hommes. Ainsi, on passe respectivement entre les deux moments de mesure de $25 \%$ à $6 \%$ de jeunes travailleurs déclarant un revenu mensuel inférieur ou égal à 930 euros $^{4}$ net. Dans le groupe féminin, les pourcentages respectifs sont de $59 \%$ puis $47 \%$. À l'autre bout de l'échelle des revenus, près de $3 \%$ de travailleurs masculins gagnaient plus de 1363 euros net au premier moment d'observation. Ils étaient plus de $22 \%$ dans cette situation trois ans plus tard. On ne trouve aucune femme de notre échantillon dans cette catégorie de revenu, ni au temps 1 ni au temps 2. Les salaires des femmes sont donc rapidement distancés par les salaires des hommes. De plus, ils sont toujours aujourd'hui moins liés que ceux des hommes au niveau de diplôme atteint, même après contrôle de la variable de travail à temps plein ou temps partiel.

\section{L'IMPACT DIFFERENCIÉ DES RAPPORTS SOCIAUX DE SEXE DANS LA SPHÈRE PRIVÉE}

De manière traditionnelle, les différences sexuées au niveau des carrières professionnelles sont attribuées à la charge familiale et aux choix de vie des seules femmes. La théorie du genre nous invite à revisiter ce point de vue, à comparer les transitions démographiques effectuées par les hommes et les femmes, les moments où ces changements interviennent dans la vie des personnes. Elle incite également à tenter de comprendre les enjeux sexués de ces temporalités, leurs impacts en matière de développement de la trajectoire professionnelle. C'est ce que nous faisons dans la deuxième partie de cet article. Nous regardons en quoi les engagements de couple et de parent s'articulent de manière sexuellement marquée avec le démarrage des carrières professionnelles. À ce niveau, nous interrogeons le phénomène toujours

${ }^{4}$ À noter que la monnaie nationale, le franc belge, était toujours de mise durant les périodes étudiées. 
présent de l'homogamie sociale ${ }^{5}$ au sein des jeunes couples. Enfin, nous questionnons l'importance accordée par les travailleurs et travailleuses à l'exercice de leur profession et l'affirmation répandue selon laquelle les hommes ont tendance à sacrifier leur vie privée à leur profession tandis que les femmes, moins ambitieuses à ce niveau, favorisent leur projet familial.

\section{Les rapports entre transitions professionnelles et familiales diffèrent selon le groupe sexué}

De nombreuses recherches ont mis en avant l'articulation existant entre les différentes séquences d'engagements démographiques et professionnels (Galland, 1996).

Elles relèvent conjointement :

- un âge plus précoce pour les femmes que pour les hommes en ce qui concerne la mise en couple, l'officialisation d'une union ou l'arrivée du premier enfant ; - une tendance actuelle des jeunes des deux sexes à retarder leurs engagements de conjoint et de parent. Nos données permettent de confirmer et d'illustrer ces tendances. Le tableau 4 montre une différence significative entre les situations de vie des hommes et des femmes au second temps d'observation.

La tendance féminine à la précocité des engagements reste vraie pour chaque niveau de diplôme, même si, parmi les femmes, les plus diplômées s'avèrent les

${ }_{5}^{5}$ C'est-à-dire la tendance des personnes à rechercher un compagnon qui corresponde à son niveau social ou culturel. moins pressées à s'engager en tant que conjointe ou que mère. Ces dernières jouent un rôle important dans le mouvement de diversification des trajectoires démographiques et familiales. En fait, retarder ses engagements de conjointe et surtout de mère représente une stratégie démographique souvent nécessaire pour la jeune femme désireuse d'asseoir son positionnement professionnel ${ }^{6}$. Les hommes, de leur côté, restent plus libres dans leur choix, même dans une phase de lancement de leur carrière ; ils se sentent toujours moins concernés par l'articulation entre les sphères et sont objectivement moins contraints par ces dernières 7 . Leurs engagements familiaux de conjoint et de parent ne s'accompagnent pas autant que pour les femmes de contraintes d'organisation quotidienne. Les jeunes hommes comptent le plus souvent sur leur compagne pour régler ces « questions » (par exemple : trouver une solution de garde pour l'enfant à naître, pour le nourrisson malade ou

\footnotetext{
${ }^{6}$ D'autres enquêtes menées parallèlement à notre analyse sur les données Panel confirment l'existence de telles stratégies, pas toujours conscientes, de la part des femmes qui se doivent de relever le challenge d'ascension sociale après l'obtention d'un diplôme élevé. $C f$. également l'article d'Armelle Testenoire dans le présent numéro.

${ }^{7}$ Significativement plus de femmes que d'hommes affirment déjà, 5 ans après la sortie des études, que des obligations familiales hypothèquent le déroulement de leur carrière. Par ailleurs, l'enquête interroge tous les adultes, d'un côté quant à leur participation concrète aux tâches domestiques, parentales, de solidarité sociale et intergénérationnelle, d'un autre côté quant à l'aide reçue des parents dans l'organisation du quotidien. Les hommes peuvent toujours plus compter sur l'aide féminine (de leur mère, de leur conjointe) pour se décharger des tâches et obligations familiales. Entre les deux moments de mesure, la répartition sexuelle de ces tâches est redevenue plus inégalitaire. Au sein des couples constitués de manière précoce, la participation masculine décroît quantitativement et l'homme reprend le contrôle de différents postes « clés » pris en charge précédemment par la femme. C'est le cas de la gestion du budget et de l'épargne.
}

Tableau 4

Répartition des sujets par situation de vie, cinq ans après la fin des études (au temps 2)

\begin{tabular}{|l|c|c|c|}
\hline & \multicolumn{3}{|c|}{ Situation de vie au temps 2 } \\
\hline & Hommes & Femmes & Total \\
\hline En couple, avec enfant(s) & $20 \%$ & $33 \%$ & $25 \%$ \\
\hline En couple, sans enfant & $29 \%$ & $33 \%$ & $31 \%$ \\
\hline Pas en couple, avec enfant(s) & $0 \%$ & $8 \%$ & $41 \%$ \\
\hline Pas en couple, sans enfant & $51 \%$ & $26 \%$ & \\
\hline & \multicolumn{2}{|c|}{ Différence significative entre les sexes au seuil de $7 \%$} \\
\hline
\end{tabular}

PSBH, sous-échantillon total 
l'écolier en congé). C'est elle qui adapte le plus souvent ses horaires et son temps de travail, mobilise son réseau familial ou social quand le besoin s'en fait sentir. Enfin, c'est surtout elle qui assume la charge mentale de la famille. À propos des rôles sexués, Daune-Richard (1993) indique avec justesse que ce n'est pas seulement la charge concrète, ponctuelle et mesurable de travail domestique qui discrimine mais la question de savoir qui a la famille dans la tête, et ce y compris de manière proactive. Ce dernier point est important car il indique en quoi les normes sociales intériorisées par les femmes de manière progressive et précoce peuvent intervenir pour réduire leurs prétentions professionnelles, même avant que les problèmes concrets d'organisation ne voient le jour. Ainsi, une majorité de femmes et d'hommes valorisent l'opportunité, pour une mère, de travailler à temps partiel ${ }^{8}$.

Au masculin comme au féminin, les étapes tant professionnelles que familiales ne s'inscrivent pas au hasard dans l'univers social. En même temps, chez les jeunes hommes et chez les jeunes femmes, l'engagement de couple ne s'articule pas de la même manière à l'engagement professionnel (Blöss et al., 1995), et ce sans qu'on puisse déduire nécessairement un effet unilatéral de la sphère familiale sur la sphère professionnelle. Ainsi pour les hommes, des difficultés d'insertion professionnelle peuvent hypothéquer la mise en couple ; à l'opposé, $85 \%$ des femmes hors emploi au temps 1 se retrouvent en couple au temps 2, contre moins de $50 \%$ dans le groupe initialement en emploi.

Par ailleurs, une cohabitation précoce influence faiblement le rapport à l'emploi ultérieur des hommes, alors qu'elle va augmenter la propension des jeunes femmes à se retrouver salariées à temps partiel, cela sans que l'accès à l'emploi s'en trouve néanmoins désormais affecté. Une évolution historique est visible ici dans la mesure où, au siècle passé, une pression sociale forte s'exerçait à l'encontre du travail des femmes mariées et des mères ${ }^{9}$. Être mère

\footnotetext{
${ }^{8}$ Attention, cela ne signifie pas que toutes les femmes qui ont un travail a temps partiel l'ont souhaité ou l'ont accepté en fonction d'impératifs familiaux, loin de là, comme nous l'avons mentionné. Cette affirmation exprime dans l'absolu à la fois le besoin des adultes de disposer de temps pour s'occuper des enfants et la norme sociale qui veut que ce rôle soit dédié en priorité aux mères. ${ }^{9}$ Pour M. Maruani (1996), les discriminations sexuées se déplacent, prennent une autre forme. Elles se situent aujourd'hui principalement au niveau de la qualité de l'emploi et du parcours professionnel.
}

rapidement après la sortie des études (et en tout cas au temps 1) va affecter à la fois et les chances de maintien en emploi au temps 2 (47\% des femmes concernées contre $92 \%$ de l'autre groupe sont dans ce cas) et celles d'occuper en fin de période un emploi à temps plein et en CDI (seules $29 \%$ des mères au temps 1 se retrouvent dans ce cas).

Ainsi, la parentalité constitue un élément important à partir duquel les inégalités de genre continuent à se construire. Au total, ce sont bien les sujets sans enfant, hommes et femmes qui, au temps 2, voient exploser leur temps de travail (durée moyenne supérieure à 41 heures) et qui démarrent une carrière, et cela assez indépendamment du niveau de diplôme. À l'inverse, au sein du groupe de parents, seules les femmes voient leur engagement professionnel réduit en termes de temps de travail, et ce le plus souvent indépendamment de leur propre volonté.

De manière schématique, les femmes les moins qualifiées se retrouvent les plus soumises aux aléas de l'offre d'emploi, à l'instabilité professionnelle, au sousemploi et au risque de retrait prématuré du marché du travail. La plupart des emplois non qualifiés pour les jeunes femmes se situent dans des secteurs où les contrats à durée déterminée et à temps partiel sont fréquents. Au deuxième moment d'observation, seule la moitié des mères détentrices au maximum d'un diplôme de l'enseignement secondaire occupent un emploi rémunéré; et ce, alors que les perspectives professionnelles du partenaire restent, de plus en plus souvent, incertaines elles aussi et que le besoin d'un second emploi se fait le plus souvent cruellement sentir.

De leur côté, les mères les plus diplômées « résistent » dans l'emploi, mais bon nombre d'entre elles sont contraintes de revoir à la baisse leur projet de développement professionnel; il s'agit de permettre implicitement au partenaire de faire décoller rapidement sa carrière, et pour cela, de le libérer des entraves liées à la gestion du temps et des distances. C'est plus souvent la femme qui adapte progressivement ses exigences, son temps et son statut de travail en fonction de la situation professionnelle de son partenaire, plutôt que l'inverse ${ }^{10}$. Dans les

\footnotetext{
${ }^{10}$ On trouve plus qu'avant des schémas inversés où la carrière de la femme prime mais la règle selon laquelle une carrière " haute " s'appuie sur une plus «basse » reste de toute façon d'actualité.
} 
réponses aux questions portant sur leur niveau de satisfaction, leurs attitudes et leurs valeurs (Gavray, 2003), on peut voir la nécessité qu'éprouvent certaines conjointes très diplômées de justifier positivement ce changement de cap. Cette attitude renvoie à leur besoin de maintenir une bonne «image de soi ». À ce propos, Gerris (1995) a montré l'énergie déployée par les mères diplômées chargées de préparer au mieux leurs enfants en fonction d'un avenir compétitif (par l'apprentissage des langues, une diversité de découvertes culturelles, la pratique du sport...) ; cela demande de leur part des compétences de plus en plus ciblées et spécialisées. Les données montrent néanmoins que seul un petit nombre de femmes qualifiées réinvestissent à plein temps leur rôle traditionnel d'organisatrice du foyer. La plupart d'entre elles, engagées dans un processus d'autonomisation et plus sensibles aux injonctions de réussite et d'indépendance (Gavray, 2004b), veulent à tout prix garder un emploi mais vont accepter ou viser, durant la période où ce sera nécessaire, des conditions de travail leur laissant plus de marge de manœuvre pour mener de front leurs obligations familiales et professionnelles. À l'issue des cinq années qui suivent la sortie des études, $38 \%$ des femmes en couple, contre aucun homme, affirment que les soins et l'attention qu'ils ont apportés à d'autres personnes ont pesé sur le démarrage de leur carrière professionnelle.

Nous pouvons illustrer le renforcement de l'enjeu lié au temps au sein des couples au fur et à mesure que le temps passe. Au temps 1, plus le nombre d'heures de travail effectué est élevé chez un partenaire, plus il l'est également chez l'autre; la proximité des opportunités professionnelles des conjoints reflète la persistance d'une certaine homogamie culturelle et éducative au sein des couples. Trois ans plus tard, plus le temps de travail des hommes est important, plus le temps de travail des femmes diminue. La femme se trouve de manière concomitante confrontée à la rigidification des rôles sexués ${ }^{11}$, nous l'avons déjà mentionné.

Les enjeux autour du temps nécessaire à l'organisation familiale se renforcent au fur et à mesure que l'ensemble

\footnotetext{
${ }^{11}$ Par exemple, au temps 2, aucun homme en couple mais $59 \%$ des jeunes femmes affirment prendre en charge la totalité de la tâche de nettoyage. Au temps 1 , ces pourcentages étaient de $3 \%$ et de $42 \%$.
}

des engagements adultes se précisent. Les analyses nous montrent néanmoins que le scénario d'une position professionnelle moyenne des deux partenaires s'accompagnant d'une durée de travail proche de la moyenne générale semble favoriser à son tour un partage plus équilibré des tâches familiales et éducatives entre l'homme et la femme (Gavray, 2003). Cette dynamique favorable semble encore facilitée par une plus grande proximité de revenus, de profession et de statut de travail entre les partenaires ${ }^{12}$. La marche vers l'égalité serait donc aujourd'hui le fait de citoyens situés au centre de l'échelle socioprofessionnelle, et non pas au sommet, comme le laisserait penser le résultat de certains sondages centrés sur le discours des personnes ${ }^{13}$.

\section{L'homogamie sociale influence les inégalités entre les sexes, entre les femmes et entre les groupes sociaux}

Les données confirment que le travailleur n'est pas un isolé au travail. Sa position professionnelle est tributaire des caractéristiques de son groupe familial mais aussi du profil de son conjoint. Le tableau 5 confirme au temps 2 (cinq ans après la sortie des études) un meilleur équilibre de la durée hebdomadaire de travail entre les conjoints quand l'homme détient un diplôme de niveau moyen. Dans un même temps, les écarts-type nous fournissent d'autres indications : les écarts moins importants concernant ce groupe par rapport aux deux autres (et cela pour les deux sexes) indiquent en son sein une plus grande proximité de situation en matière de temps de travail. On observe une palette plus variée de temps de travail dans le groupe féminin que masculin.

À y regarder de plus près, le niveau de diplôme masculin le plus élevé, comme le plus bas, creuse l'écart sexué en matière de durée de travail au sein du

\footnotetext{
${ }^{12}$ On note que le fait que les deux partenaires exercent un emploi dans le domaine de la santé ou de l'éducation favorise encore le partage égalitaire (probablement en raison d'une sensibilité supérieure des hommes au travail de soins - notion de «care» dans l'univers anglo-saxon - taxé de féminin mais dont ils sont plus proches via leur pratique professionnelle, de même que par les aménagements horaires réalisés pour se relayer auprès des enfants ; ces aménagements sont justifiés et facilités par ce type d'emploi). ${ }^{13}$ Ces sondages indiquent que les hommes les plus diplômés sont le plus d'accord avec le principe d'égalité sexuée et la nécessité de sa mise en application (Gavray, 2004).
} 
Tableau 5

Heures de travail hebdomadaires au temps 2 effectuées par chaque membre du couple, en fonction du niveau de diplôme de l'homme

\begin{tabular}{|c|c|c|}
\hline Quand diplôme maximum masculin = enseignement secondaire \\
\hline & Nombre moyen d'heures de travail & Ecart-type \\
\hline Homme & 42 & 7.1 \\
\hline Femme & 37 & 7.6 \\
\hline Quand diplôme maximum masculin = enseignement supérieur de type court \\
\hline \multicolumn{2}{|c|}{ Nombre moyen d'heures de travail } \\
\hline Homme & 40 & Ecart-type \\
\hline Femme & 40 & 3.6 \\
\hline Quand diplôme maximum masculin $=$ enseignement supérieur de type long \\
\hline \multicolumn{2}{|c|}{43} & 5.8 \\
\hline Homme & Nombre moyen d'heures de travail & Ecart-type \\
\hline Femme & 38 & 8.1 \\
\hline
\end{tabular}

PSBH, analyse restreinte aux couples de salariés au temps 2 (5 ans après la sortie des études) et effectuant au moins 15 heures hebdomadaires de travail par semaine.

Exemple de lecture : lorsque le conjoint est diplômé au plus de l'enseignement secondaire, il travaille en moyenne 42 heures (+/- 7,1 heures) et la femme 37 heures (+/- 7,6 heures).

couple, comme d'ailleurs la probabilité d'emploi à temps partiel pour la femme. En raison de la persistance de l'homogamie sociale au sein des couples, le groupe le moins diplômé doit faire face à l'offre partielle d'emploi faite aux femmes. Cet emploi est pourtant le plus souvent strictement nécessaire au bien-être du ménage. Le risque de précarité qui en découle pour le groupe familial atteint le moral des femmes, plus que leur mauvais positionnement professionnel personnel (Born et Gavray, 1996). C'est l'inverse pour les hommes. Cette construction différente de l'identité est attribuable aux normes intériorisées au cours du processus de socialisation. Elle renforce à son tour les rôles sexués traditionnels, encore plus dans ce groupe social.

Au sein du groupe le plus diplômé, la carrière masculine continue à dominer, ce qui engendre à terme un certain sentiment d'insatisfaction professionnelle et de malaise psychosocial ${ }^{14}$ chez les femmes diplômées; du moins chez celles qui estiment qu'elles

${ }^{14}$ Des questions sont posées dans le questionnaire sur le niveau de satisfaction général et relatif à chacune des sphères de la vie (professionnelle, familiale, sociale...). Une échelle de dépression (de Moos) a été également incluse. gâchent leurs chances et que le fait de revoir leurs prétentions professionnelles initiales à la baisse est tout sauf un réel choix. A priori, ces femmes diplômées disposent des ressources sociales, éducatives et professionnelles les plus favorables, mais elles se sentent tiraillées entre des injonctions contradictoires et difficilement gérables d'un point de vue subjectif. Il est intéressant de remarquer que les jeunes filles en ascension sociale par rapport à leurs parents sont plus combattives pour défendre leur propre carrière.

En fait, si ce sont les jeunes femmes les plus diplômées qui se montrent les plus « en questionnement» par rapport à leur situation, dans le groupe masculin, ce sont les sujets cette fois-ci les moins diplômés qui s'avèrent les moins satisfaits de leur situation, qui doutent le plus de leur valeur et de leur avenir ${ }^{15}$. Leur capacité à jouer leur rôle de pourvoyeur de revenus et à assurer la qualité de vie de leur famille dans la durée

\footnotetext{
${ }^{15}$ Des articles récents foisonnent dans la presse sur la perte d'identité des jeunes hommes, cette dernière étant souvent attribuée à une ivresse de pouvoir des femmes. L'approche de genre nous invite à comprendre en quoi et pourquoi un mode de fonctionnement sociétal et institutionnel en décalage par rapport à ses principes fondateurs (comme le principe égalitaire entre les sexes) atteint de manière conjointe mais différente l'image de soi des hommes et des femmes.
} 
est remise en cause (plus forte exposition à l'emploi temporaire et aux revenus professionnels bas, moindres opportunités de promotion que précédemment).

On voit en quoi le genre continue à entretenir la vision des rôles traditionnels, tant dans le groupe féminin que masculin, et comment la résistance de normes sexuées rigides dans un monde en transformation, où le principe d'égalité ne se traduit pas nécessairement au niveau des fonctionnements institutionnels (Gadrey, 2001), peut affecter subjectivement différents groupes de populations.

Néanmoins, le sentiment de malaise des femmes diplômées devant un marché du travail fonctionnant selon des normes masculines ${ }^{16}$, de même que les difficultés matérielles des moins diplômées et les discriminations professionnelles qu'elles vivent ${ }^{17}$ n'induisent pas nécessairement un comportement de victime n'ayant aucune prise sur son avenir et sur l'évolution du contexte. Les nouveaux enjeux qui se dessinent vont conduire certaines femmes à initier du changement, et ce tout d'abord dans leur univers de vie le plus proche, au risque de confrontations dans le couple, voire de rupture ${ }^{18}$. D'autres recherches que nous avons menées sur une population plus âgée (Gavray, 2003) montrent comment, à l'occasion d'une rupture ou d'une remise en couple, les femmes vont être plus attentives à leur autonomie, redéfinir leurs exigences au niveau du fonctionnement de couple, mais aussi défendre un projet professionnel de meilleure qualité. Les projets des individus jouent un rôle important sur l'évolution de nos sociétés modernes car ils servent de variable intermédiaire autour de laquelle se réorganisent continuellement les attitudes sur le terrain et au-delà les modèles normatifs.

Ces différents résultats nous montrent bien, premièrement, que la question de l'insertion professionnelle est

\footnotetext{
${ }^{16}$ Où l'engagement professionnel prime sur les autres engagements. ${ }^{17}$ Les femmes non diplômées représentent le groupe disposant du pouvoir de négociation le plus faible et le plus exposé au sousemploi et aux moins bonnes conditions de travail. La dualisation du marché de l'emploi les relègue dans l'emploi secondaire (Broze et al., 2002).

18 Dans ce contexte, l'augmentation des risques de rupture de couple à l'initiative des femmes relatée dans les analyses démographiques récentes (Blöss et al., 1994) ne provient pas d'abord de l'avancée de leur autonomie financière mais résulte davantage d'exigences grandissantes de partage et d'équité non satisfaites.
}

liée aux autres sphères d'insertion; deuxièmement, que l'insertion comporte une dimension objective et subjective inséparables l'une de l'autre; troisièmement, que des mouvements contradictoires sont continuellement en tension (le premier conservateur, le second novateur) et font évoluer les problématiques et les enjeux à tous les niveaux. Les pages suivantes vont encore nous permettre d'illustrer ce point.

\section{L'importance accordée à l'emploi par les hommes et les femmes n'est pas figée}

L'importance accordée à l'emploi change avec les générations mais aussi, chez les mêmes personnes, au fur et à mesure qu'elles avancent dans leur cursus de vie. De nombreux travaux psychosociologiques récents ont montré que la jeune génération, (sousentendu ici la population de référence masculine et diplômée), relativiserait davantage son engagement professionnel, désirant consacrer du temps à d'autres activités et engagements (Fournier, 2000). Des recherches plus ciblées montrent deux types de réactions opposées de la part des jeunes hommes en difficulté d'insertion. Certains s'attribuent toute la responsabilité de leur insertion professionnelle et sociale et survalorisent l'emploi. À l'inverse, d'autres en viennent à désinvestir précocement ce domaine devant leur extrême difficulté à obtenir leur statut social d'homme adulte. En outre, on remarque que les projets de vie des jeunes femmes, aujourd'hui plus diplômées que les hommes, restent plus diversifiés (Nicole-Drancourt,1990). De manière générale, les femmes seraient conscientes que leur autonomie passe avant tout par l'exercice d'une activité professionnelle rémunérée (Maruani, 1996) et elles se mobiliseraient dans ce sens.

Ces différents résultats montrent que l'implication subjective dans l'exercice de son travail, la place que l'on attribue à l'exercice de sa profession dépendent des différents bénéfices objectifs et subjectifs retirés ou escomptés. Conjointement se confirme un phénomène mis en lumière par Pierre Bourdieu : les différents groupes de population disposant du pouvoir de négociation le plus faible (dont les jeunes et les femmes) sont amenés, jusqu'à un certain point, à partager le point de vue, les normes et les modes de fonctionnement imposés par le « dominant». 
Tableau 6

Type d'investissement professionnel (en \%)

\begin{tabular}{|c|c|c|c|c|c|c|}
\hline & \multicolumn{3}{|c|}{ 1,5 ans après la fin des études (Temps 1) } & \multicolumn{3}{|c|}{5 ans après la fin des études (Temps 2) } \\
\hline & Hommes & Femmes & Total & Hommes & Femmes & Total \\
\hline $\begin{array}{l}\text { Investissement } \\
\text { professionnel } \\
\text { inconditionnel }\end{array}$ & 33 & 8 & 23 & 29 & 0 & 12 \\
\hline $\begin{array}{l}\text { Investissement } \\
\text { relatif }\end{array}$ & 63 & 92 & 73 & 71 & 94 & 90 \\
\hline \multirow[t]{2}{*}{$\begin{array}{l}\text { Investissement } \\
\text { "obligé " }\end{array}$} & 5 & 0 & 3 & 9 & 6 & 8 \\
\hline & \multicolumn{3}{|c|}{ Différence significative entre les sexes } & \multicolumn{3}{|c|}{ Différence significative entre les sexes } \\
\hline
\end{tabular}

PSBH, analyse restreinte aux sujets du sous-échantillon travailleurs au temps 1, puis au temps 2

Seuil de significativité inférieur à $1 \%$.

Lecture : le tableau 6, qui concerne toujours notre sous-échantillon de départ, classe les travailleurs masculins et féminins aux deux dates d'observation, cela à partir de la réponse à une question portant sur l'importance que revêt leur emploi. Ils avaient le choix entre affirmer soit qu'ils étaient prêts à faire de grands sacrifices pour leur carrière professionnelle, soit qu'ils étaient attentifs à doser leur implication professionnelle de sorte que leurs autres engagements n'en souffrent pas, soit encore que, s'ils n'étaient pas obligés de travailler, ils s'arrêteraient.

Nos données montrent ainsi un surinvestissement professionnel plus courant dans le groupe masculin et une tendance des hommes et des femmes, le temps passant, à relativiser davantage l'importance accordée à l'exercice de leur profession; plus de sujets désinvestissent radicalement cette dernière, même si leur proportion reste faible.

Les résultats présentés dans le tableau 6 sont intéressants dans la mesure où ils montrent que, le temps passant, une large majorité des femmes mais aussi un nombre croissant d'hommes de la population étudiée relativisent leur engagement professionnel. Les données confirment que cette position moyenne est adoptée de manière préférentielle par les hommes très diplômés qui effectuent un nombre d'heures hebdomadaires de travail particulièrement élevé. Nous avons néanmoins vérifié que, dans la pratique, ces personnes placent leur travail en première position et peinent à accorder une place de choix aux autres engagements. Nous voyons clairement que le genre agit conjointement sur les femmes et sur les hommes, ces derniers se trouvant dans les faits peu autorisés, eux aussi, à mieux équilibrer les différentes composantes de leur vie adulte.

La survalorisation de l'implication professionnelle concerne à la fois des sujets qualifiés comme des sujets peu qualifiés, aux perspectives professionnelles incertaines, ces derniers misant sur l'effort personnel pour sortir du lot.
Au total, les femmes affirment moins souvent que les hommes travailler par stricte obligation. Les différents résultats présentés contredisent la croyance selon laquelle les femmes s'impliqueraient moins dans leur emploi que les hommes et feraient par exemple correspondre leur décision d'activité à un simple calcul économique automatique (en fonction des revenus du mari ou après calcul financier comparatif entre des décisions alternatives). En fait, les femmes se trouvent historiquement à une autre étape de leur processus d'autonomisation, l'identité féminine passant désormais principalement par la participation au travail rémunéré. La conjonction de ces deux phénomènes peut constituer un désavantage pour les femmes, dans la mesure où la conjoncture d'emploi est peu favorable mais aussi où le marché du travail peut utiliser ces arguments et la faiblesse du pouvoir de négociation qui en découle pour maintenir les femmes dans un segment de l'emploi moins valorisé et moins valorisant. Ce contexte, plutôt que la nature biologique ou les difficultés familiales des femmes, peut pousser ces dernières à penser l'emploi en termes d'insertion plutôt que de carrière, à miser avant tout sur la qualité relationnelle du travail et sur la fidélité à l'employeur, à s'accrocher à un emploi pour lequel elles se disent surqualifiées. Ces attitudes ont pu être vérifiées dans notre enquête. 
À ce stade de notre investigation, il nous semble inapproprié de vouloir rapporter les différences sexuées de trajectoire professionnelle à des décisions strictement personnelles et familiales, touchant uniquement la sphère privée.

\section{LES DÉBUTS DE PARCOURS MARQUENT LA SUITE DE LA CARRIÈRE}

Dans quelle mesure la moindre qualité et sécurité d'emploi de départ à laquelle peuvent prétendre les femmes contribuent-elles à expliquer les écarts grandissants entre les débuts de trajectoire professionnelle des hommes et des femmes ? C'est la question qui nous occupera dans ce chapitre. Dans un premier temps, nous étudierons les transitions de positions socioprofessionnelles. Dans un second temps, nous examinerons les situations d'arrivée cinq ans après la fin des études en fonction d'une combinaison de variables rendant compte à la fois de la situation familiale des personnes et de leur statut d'emploi antérieur.

\section{Les positions et les transitions de départ construisent la dynamique $d^{\prime}$ insertion}

À côté des différents facteurs personnels, objectifs et subjectifs, pouvant expliquer des différences sexuées en matière de qualité de début de trajectoire professionnelle, un autre facteur mérite de retenir notre intention. C'est celui de la qualité des premiers emplois auxquels les sujets accèdent dans un parcours d'insertion plus long et discontinu qu'avant (Rose,1998). Les données PSBH nous fournissent une approche longitudinale utile pour visualiser les enchaînements et transitions. Les données confirment que $30 \%$ des travailleurs initialement en emploi atypique ${ }^{19}$ se retrouvent en emploi à temps partiel trois ans plus tard, alors qu'aucun sujet n'est dans ce cas dans le groupe initialement en emploi typique. Par ailleurs, le chômage et le travail

${ }^{19}$ Tel que défini au point 1.2. (« Les écarts de position augmentent cinq ans après la fin de la scolarité »). atypique se conjuguent, comme attendu, pour augmenter significativement les risques de sortie d'activité (Gavray, 2003). Ainsi, détenir un emploi à temps partiel ou atypique au départ augmente les probabilités de se retrouver hors emploi au second moment d'observation. La relation inverse se vérifie également. Si une telle dynamique fragilisante vaut pour les deux groupes sexués, les jeunes hommes restent au final très peu concernés par les deux phénomènes en interaction. Au total, le début de trajectoire professionnelle féminine se trouve plus souvent interrompu. Au deuxième temps de mesure, $30 \%$ des femmes contre $20 \%$ des hommes ont connu une période de chômage avant d'accéder à l'emploi exercé. Par ailleurs, sur les 48 mois précédant ce moment d'observation, pour $11 \%$ des hommes mais $21 \%$ des femmes, le nombre de mois hors emploi est supérieur au nombre de mois en emploi.

Nos résultats montrent que, pour les femmes, ce n'est pas exactement la même dynamique de facteurs qui va expliquer, d'un côté l'accès à l'emploi, de l'autre la qualité de l'emploi occupé. Le niveau de diplôme ressort pour les femmes comme « le » passeport le plus important pour l'emploi. En revanche, la qualité de l'emploi occupé cinq ans après la fin des études s'avère liée de manière moins forte et moins directe au niveau de diplôme. Au total, il s'avère qu'ici ce n'est plus tant le niveau de diplôme que la rentabilisation précoce de ce dernier dans un emploi de qualité qui donne une impulsion à la trajectoire d'emploi féminine, évitant plus facilement la remise en cause ou la stagnation ultérieure de cette dernière, quand les pressions extérieures et familiales se précisent. Quel que soit le niveau de diplôme, la détention directe ou rapide d'un emploi typique diminue les probabilités de non-emploi en fin de période d'observation et augmente les chances d'un bon positionnement.

\section{Les facteurs familiaux et professionnels construisent les opportunités}

D'autres résultats viennent encore confirmer et illustrer l'impact que peut jouer la qualité des premiers pas d'insertion professionnelle sur la suite du processus : 
À partir de modèles de régression multiple ${ }^{20}$ qui testent par étapes successives l'impact d'un ensemble de variables de plus en plus restreint, on arrive au résultat intéressant selon lequel, au terme de cinq ans (temps 2), le positionnement professionnel atypique de la femme en couple très diplômée dépend finalement, et dans un sens positif, de deux facteurs articulés: le temps de travail du partenaire, facteur évoqué précédemment, mais aussi la qualité médiocre de l'emploi antérieur.

Nous avons pu vérifier que tant le type d'emploi occupé au début de la trajectoire d'emploi que le nombre et les temporalités des passages entre emploi et non-emploi se révèlent avoir une importance sur la suite $\mathrm{du}$ processus d'insertion professionnelle féminin.

La durée hebdomadaire habituelle de travail dans le groupe féminin au temps 2 est, quant à elle, corrélée négativement et à la maternité (moyenne de 36 heures de travail chez les femmes non concernées et de 31 heures pour les mères) et à la détention préalable d'un emploi atypique au premier moment d'observation (écart de 4 heures de travail entre les deux situations).

Si de son côté la durée moyenne de travail masculine est reliée à l'expérience de paternité, elle va ici augmenter entre les moments d'observation, et cela sans qu'interviennent les enchaînements successifs de statuts d'emploi. Un résultat apparaît ici comme particulièrement intéressant: dans le groupe masculin, le temps de travail est accru avant tout par les opportunités de décollage de carrière dans ses aspects financiers et promotionnels : entre en jeu le fait que le travailleur a obtenu un poste d'encadrement, tandis que le niveau de diplôme ne joue pas.

Se profile ici une différence déjà mise en avant par les théoriciennes du genre selon laquelle, toujours de nos

\footnotetext{
${ }^{20}$ L'analyse en régression multiple « stepwise » permet d'identifier les prédicteurs les plus puissants - parmi un ensemble de prédicteurs potentiels. Si un prédicteur n'est pas retenu, cela signifie que sa prise en compte n'ajoute rien à la prédiction de la variable une fois que l'on tient compte des prédicteurs retenus. Vous trouverez une illustration détaillée des méthodes et modèles utilisés dans le rapport de recherche «Violence à l'école : enquête de victimisation dans l'enseignement secondaire de la Communauté française de Belgique », (2003), sur le site http://www.ulg.ac.be/psydel/
}

jours et de manière générale ${ }^{21}$, les femmes ont un emploi tandis que les hommes font carrière. Nos données confirment la présence des rapports sociaux de sexe dans l'ensemble des dimensions étudiées ; ce constat invite à ne pas considérer le sexe comme une variable parmi d'autres dans les modèles explicatifs testés (Broze et al., 2002).

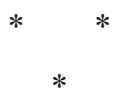

Les inégalités sexuées n'ont pas disparu au sein de la jeune génération. Certaines persistent sous des formes anciennes, tandis que d'autres se reconstruisent sous des formes renouvelées. Ainsi, un rapprochement constaté au niveau des taux d'activité, d'emploi et de chômage, à un moment proche de la sortie des études, ne suffit nullement à confirmer un mouvement d'égalisation des positions et des chances de carrière entre les jeunes hommes et les jeunes femmes.

Par ailleurs, si un effet de la sphère familiale sur la sphère professionnelle existe bien pour les deux sexes, il s'exerce de manière le plus souvent opposée et reste conditionné au contexte d'emploi dans lequel il s'inscrit. Ce contexte nous semble trop peu souvent intégré dans les analyses ; cela s'avère d'autant plus dommageable que l'on se trouve aujourd'hui face à des transformations structurelles importantes du marché du travail et du fonctionnement des entreprises. Les résultats de notre recherche nous laissent penser que le marché du travail en recomposition s'appuie sur des normes sexuées traditionnelles, au détriment des carrières professionnelles d'une partie non négligeable de jeunes femmes. De nos jours, ce n'est pas tant le niveau de diplôme que la rentabilisation précoce de ce dernier dans un emploi et un début de trajectoire professionnelle de qualité qui donnent

\footnotetext{
${ }^{21}$ Notons que des recherches récentes tentent de s'éloigner d'une approche en termes de moyennes pour s'intéresser aux cas singuliers, qui font exception. Cette approche est intéressante dans la mesure où elle nous instruit sur les conditions favorisant la résilience, terme employé par le neuropsychiatre B. Cyrulnic pour signifier la capacité de «s'en sortir », de rebondir dans des conditions peu favorables. Néanmoins, la taille de l'échantillon ne nous permet pas de creuser cette piste. Par ailleurs, la contradiction n'est qu'apparente entre la réussite professionnelle de certaines femmes et l'insécurité professionnelle grandissante pour une large part des
} travailleuses. 
une impulsion à la trajectoire d'emploi féminine, évitant plus facilement la remise en cause ultérieure de cette dernière quand les pressions extérieures et familiales se précisent.

Dans l'articulation entre sphère privée et sphère publique, le temps ressort comme un enjeu social central. Par sa rareté, il exerce une pression importante sur la vie des personnes et des familles. Il entrave aussi la réalisation de plus d'égalité sexuée dans une organisation familiale ou le soin d'autrui est surtout la responsabilité des femmes. Il ne permet pas non plus une diversification progressive des sphères d'engagement et d'épanouissement des travailleurs masculins. Les opportunités précoces favorables en matière d'insertion professionnelle ou de négociation de couple autour des questions de travail et d'emploi peuvent parfois infléchir le poids de la sphère familiale pour les femmes ; mais cela se réalise souvent au prix d'un stress élevé. C'est la conséquence des tensions vécues en matière d'affectation du temps mais aussi du décalage existant entre les principes égalitaires intériorisés et une réalité inégalitaire. Les politiques publiques et d'entreprise discriminantes (Gadrey, 2001) sont également responsables car elles sont pensées du point de vue «masculin»; elles considèrent les responsabilités parentales et l'organisation familiale comme relevant strictement de la sphère privée et du « choix » individuel ou de couple.

Dans ce scénario, les mères ressortent fragilisées dans leur processus d'autonomisation par le travail. Leurs trajectoires professionnelles sont au rabais. Cette réalité pousse certaines jeunes femmes à reporter, voire à annuler leurs perspectives de vie en couple et de maternité. Dans le contexte de haute compétitivité qui se précise, et vu la persistance des rôles sexués traditionnels, deux situations extrêmes s'opposent dans le groupe masculin : celle des sujets peu qualifiés, qui parviennent encore tant bien que mal à s'insérer en emploi, qui vivent mal les perspectives moroses de carrière professionnelle et leur grande difficulté à jouer leur rôle de pourvoyeur de revenu familial ; celle des sujets très diplômés dont l'entreprise attend une disponibilité inconditionnelle et qui continuent à développer une identité avant tout professionnelle, même s'ils tentent de le démentir (Pochic, 2005) et s'ils sont les premiers à défendre la légitimité de l'égalité sexuée. Devant les impératifs de rentabilité et de flexibilité, il semble difficile pour les hommes de revendiquer, dans les faits, un meilleur équilibre entre vie professionnelle, familiale et développement personnel (Guichard-Claudic, 2005).

À terme, des mutations comme le mouvement de déstabilisation des familles et l'implication supérieure des pères dans la prise en charge des enfants à l'issue d'un divorce arriveront peut-être à fissurer les vieux schémas de comportements et de pensée. En attendant, il semble que les « laboratoires » de changement vers plus d'égalité se situent au centre de l'échelle sociale. Aujourd'hui, c'est finalement dans les couples dont les deux membres disposent de positions professionnelles proches et évaluées comme « moyennes » en termes de statut, de salaire, de durée de travail hebdomadaire et de secteur d'activité que le travail non rémunéré et le temps libre sont répartis de façon la plus égalitaire, notamment pour les couples d'enseignants. Dans les autres configurations de couple, les femmes doivent plus souvent composer avec les contraintes familiales. Elles parviennent, tant bien que mal et partiellement, à défendre leur autonomie et leur projet personnel.

J.-C. Kaufmann (2001) et A. Testenoire (cf. le présent numéro) analysent les évolutions en cours comme un mouvement de tension en spirale entre les éléments de structure (injonctions sociétales, évolution du marché du travail, théories économiques et de management en vogue...) et les dépassements individuels de ces contraintes (attitudes novatrices, renforcement des obligations de prise en compte du facteur de réalité, diversification des aspirations...).

L'histoire nous a appris qu'en la matière rien n'était jamais acquis. L'enjeu de genre dépasse la simple question d'équité entre hommes et femmes. Il occupe une place centrale dans la construction démocratique de l'Europe et dans la capacité dont celle-ci pourra faire preuve dans l'arbitrage entre intérêts économiques et humains. 
Barrere-Maurisson M.-A. (1992), La division sociale du travail : La vie en double, Paris, Éditions Presses Universitaires de France.

Baudelot C., Glaude M. (1989), «Les diplômes se dévaluent-ils en se multipliant?", in Économie et Statistique, $\mathrm{n}^{\circ} 225$, pp. 3-16.

Bellens W. (1996), «Le marché du travail des jeunes », in Revue du travail, $\mathrm{n}^{\circ} 24$, pp. 18-26.

Blöss T., Frickey A., Novi M. (1994), « Modes d'entrée dans la vie adulte et trajectoires sociales des femmes mariées », in Population, INED, $\mathrm{n}^{\circ}$ 3, pp. 637-656.

Born M., Gavray C. (1996), « Précarité psychosociale différentielle des hommes et femmes », Cahier de l'IPSA, $\mathrm{n}^{\circ} 19$, pp. 239-258.

Broze L., Gavray C., Ruyters C. (2002), « Dualisme, mobilité et déterminants familiaux : une analyse des transitions sur le marché du travail », in De la Croix D., Docquier F., Minguet C., Perelman S., Wasmer E. (Eds), Capital humain et dualisme sur le marché du travail, Éditions De Boeck Université, Coll. «Économie, Société et Région », pp. 51-67.

Chapoulie S. (2000), «Une nouvelle carte de mobilité professionnelle », in Économie et Statistique, n³31, pp. 25-45.

Commaille J. (1992), Les stratégies des femmes: Travail, Famille et Politique, Paris, Éditions La Découverte.

Daune-Richard A.-M. (1993), «Activité et emploi des femmes : des constructions sociétales différentes en France, au Royaume-Uni et en Suède », in Sociétés Contemporaines, $\mathrm{n}^{\circ} 16$, pp. 125-145.

Establet R. (1988), « Subversion dans la reproduction scolaire », in Revue économique, ${ }^{\circ} 1$.

Favereau O. (1989), «Marchés internes, marchés externes ", in Revue Économique, ${ }^{\circ} 40 / 2$, pp. 273328.
Fournier M. (2000), « Générations : volées, dorées, sacrifiées, in Sociétés Humaines, ${ }^{\circ} 108$.

Gadrey N. (2001), Travail et genre: approches croisées, Paris, Éditions L'Harmattan.

Galland O. (1995), «Une entrée de plus en plus tardive dans la vie adulte », in Économie et Statistique, $\mathrm{n}^{\circ}$ 283-284 (3-4), pp. 33-52.

Galland O. (1996), «L'entrée dans la vie adulte en France: bilans et perspectives sociologiques », in Sociologie et Sociétés, XXVIII (1), pp. 37-46.

Gavray C. (2003), Trajectoires professionnelles féminines: flexibilités et enjeux de genre, université de Liège, Faculté d'économie, de gestion et de sciences sociales, thèse en deux tomes.

Gavray C. (2004), «Engagements familiaux et professionnels : regards sur les projets des jeunes hommes et des jeunes femmes en fin de formation scolaire ", in Istace E., Laffut M., Plasman R., Ruyters C., Sphères privée et professionnelle, de Boeck, Université, Coll. «Économie, Société et Région », pp. 200-220.

Gerris J.R.M. (1995), Gezin: onderzoek en diagnostic, Assen, Van Gorcum.

Kaufmann J.-C. (2001), Ego, pour une sociologie de l'individu, Éditions Nathan.

Kergoat D. (1984), « Les femmes et le temps partiel : une relation complexe et multiforme au temps travaillé », in Travail et Emploi, ${ }^{\circ} 21$.

Leridon H., Villeneuve-Gokalp C. (1994), Constances et inconstances de la famille: biographies familiales des couples avec enfants, Paris, Presses Universitaires de France, INED, travaux et documents, cahiers $n^{\circ} 134$.

Marry C. (1996), « A quoi sert le diplôme ? », in Les cahiers du Mage, $\mathrm{n}^{\circ} 1$, pp. 49-56. 
Maruani M. (1996), « L'emploi féminin à l'ombre du chômage », in Actes de la Recherche en Sciences Sociales, $\mathrm{n}^{\circ} 115$, pp. 48-57.

Nicole-Drancourt C., (1990), «Organisation du travail des femmes et flexibilité de l'emploi», in Sociologie du travail, n², pp. 173-193.

Perrot. M. et al. (1999), Les femmes ou le silence de l'histoire, Paris, Flammarion.

Piore M.J. (1978), "Dualism in the labor market : a response to uncertain and flux, the case of France, in Revue Économique, n 19(1), pp. 26-48.
Pochic S. (2005), «Faire carrière: l'apport d'une approche en termes de genre », Formation Emploi $\mathrm{n}^{\circ}$ 91, juillet-septembre, pp. 26-48.

Rose J. (1998), Les jeunes face à l'emploi, Paris, Éditions Desclée de Brouwer, Coll. «Sociologie économique ».

Terrail J.-P. (1992), « Destins scolaires de sexe : une perspective historique et quelques arguments », in Population, ${ }^{\circ}$ 3, pp. 645-676.

Wijn J., Pruyer P. (2000), «Les jeunes et l'éducation : les nouveaux profils de transition », in Revue Internationale de Sciences Sociales, juin, $\mathrm{n}^{\circ}$ 164, pp. 169-182.

\section{Résumé}

\section{Au fur et à mesure qu'elles se construisent, les trajectoires professionnelles divergent entre les sexes : l'exemple belge}

\section{Claire Gavray}

L'analyse longitudinale montre un début de trajectoire professionnelle de plus en plus différencié entre les groupes sexués à mesure que le temps passe. En cause, la présence et la recomposition des rapports sociaux de sexe. Ces derniers interviennent sur l'ensemble des sphères (famille, emploi, sécurité d'existence...). D'un côté, les différences sexuées en matière de temporalités et de normes démographiques et familiales continuent à influencer le démarrage des carrières professionnelles. De l'autre, la "qualité » inégale de l'offre d'emploi adressée aux jeunes des deux sexes, sous le couvert d'arguments naturalistes, joue au désavantage des femmes. Certaines sont alors reléguées à la périphérie de la vie professionnelle. Pour les femmes, la qualification se confirme être un passeport pour l'emploi. Mais elle ne suffit pas à garantir des carrières égales. Au total, et dans la durée, les écarts de positions professionnelles se creusent entre les sexes mais également entre les femmes et entre les groupes sociaux, notamment en raison de l'homogamie sociale au sein des couples.

Mots clés

Cheminement professionnel, division sexuelle du travail, femme, relation famille-travail, Belgique Journal of Economic Literature : J16, J24 